\section{When burn-out reaches a pandemic level in gastroenterology: a call for a more sustainable work-life balance}

There is an increasing concern about 'burnout', exacerbated by the COVID-19 pandemic, across all sectors. In an excellent opinion piece in this edition Duong et al discuss the issues with a specific focus on our specialty. This includes the many risk factors, impact of COVID-19 and more importantly practical strategies to help minimise burnout and promote a more sustainable work life balance

- Avoid isolation

- Be fiscally and environmentally stable

- Refine time management and documentation skills

- Consider professional coaching and counselling

- Incorporate daily self-care practices It is well worth reading though and reflecting on. Maybe something we will learn post COVID-19 - think about 'burn-out' as an individual and as a profession-recognising it is the first step towards addressing it and resolving it (See page 440).

\section{Management of iron deficiency anaemia in secondary care across England between 2012 and 2018: a real-world analysis of hospital episode statistics}

Iron deficiency anaemia (IDA) is common - 2\%-5\% of adult men and post-menopausal women. If left untreated it can have a significant impact on quality of life particularly in patients with chronic medical disorders. Up to $10 \%$ will have an underlying gastrointestinal malignancy. In this issue Brookes et al use Hospital Episode Statistics to look at trends in IDA related acute and elective admissions between 2012 and 2018. In summary there was a $72 \%$ increase in admissions and a $68 \%$ increase in hospital spells with IDA as the primary diagnosis. Length of stay was longer for non- elective admissions. Of note the variability across the 195 clinical commissioning groups was high and widened during the study period from 11 to 55 non elective spells per $100000(2012 / 3)$ population to $18-118$ by $2017 / 8$. See figure 1 . The full interpretation of this data is complex but the authors rightly point out that there is potential to impact on this variance and increasing admission rates by service reconfiguration and improved algorithms of clinical care to reduce costs (significantly higher for non-elective patients), reduce re-admissions (higher for non-elective patients) and improve outcomes. Editor's Choice this month (See page 363).

There is an excellent accompanying commentary discussing the findings and treatment options - Striking the balance with intravenous iron: too much or never enough? (See page 361).

\section{Pancreatitis associated with azathioprine and} 6-mercaptopurine use in Crohn's disease: a systematic review

Thiopurines (Azathioprine and 6 Mercaptopurine) have proven efficacy in Crohn's Disease. Pancreatitis is a well recognised adverse effect although the effect size and morbidity is not well known. In this issue Gordon et al report a systematic review and meta-analysis to quantify the risk. 25 randomised controlled trials met the criteria for inclusion. The methodology and detailed analysis of these studies are in the paper. In summary, the risk of pancreatitis in patients receiving Azathioprine across all contexts was $3.8 \%$ (placebo $0.2 \%$ ). Number of patients treated with Azathioprine to cause pancreatitis was 36 for induction of remission and 31 for maintenance of remission. Interestingly there was no difference between 6- mercaptopurine and placebo although this, they report, as a very low certainty result due to low event numbers and low patient numbers. Most cases of pancreatitis were mild and resolved on cessation of therapy. There are useful forest plots in the paper to reinforce the findings. The lower toxicity of 6-MP warrants further investigation (See page 423).

\section{Anti-Mycobacterium paratuberculosis (MAP) therapy for Crohn's disease: an overview and update}

This is an important treatment option which patients may want to consider. In this issue Honap and colleagues discuss the evidence and practicalities. The association of Mycobacterium avium subspecies paratuberculosis (MAP) with Crohn's disease (CD) is controversial. MAP is the cause of Johne's disease (chronic granulomatous enteritis) in livestock. Patients with $\mathrm{CD}$ are more likely to have MAP present in intestinal tissues although whether this has a role in pathogenesis or is merely an innocent bystander remains unknown. The authors review the evidence for efficacy of combination antibiotics targeting MAP both as a single treatment and as an add on to conventional therapies. There is a potential vaccine. The authors summarise their own approach to treatment. The review is very balanced with discussion of evidence for and against treatment but also insight into the complexities of the methodology of trials including disease heterogeneity and confounders. The pragmatic recommendation from the authors is that Anti-MAP therapy for a 2 year period can be considered for patients with refractory disease or in patients where immune suppression is not appropriate. Close monitoring for treatment toxicity is essential (See page 397).

\section{Paediatric cholestatic liver disorders for the adult gastroenterologist: a practical guide}

This is an important topic. Many conditions (common and rare) that have their onset in childhood require lifelong medical care. Paediatric cholestatic liver disorders are a good example - particularly with the better treatment options (including transplantation) and improved long term outcome. In this issue Kelly and colleagues review the different conditions including clinical manifestations, complications and key issues for effective management. Biliary atresia is the most common $-0.5-1$ cases per 10000 live births. Early surgery is essential, before 3 months. Liver transplantation is required in many longer term 


\section{UpFront}

(most common indication for liver transplantation in a paediatric setting). Other conditions discussed include Progressive Familial Intrahepatic Cholestasis, Alagille syndrome (multiple extrahepatic manifestations) and Alpha one antrypsin deficiency. All present significant challenges with long term management. This is an authoritative update - practical and focused - and will be very helpful to teams managing children, young people and adults before and after the transition from paediatric to adult services (See page 404).

Please enjoy this issue. Please continue to read, enjoy and feedback on the journal. Follow us on twitter @ FrontGastro_BMJ and listen to our regular podcasts accessed via the journal website https://fg.bmj.com/

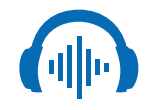

Listen to Podcast fg.bmj.com 